SLAC-PUB-9591

Nov. 2002

\title{
Limitations of Electron Beam Conditioning for Free-Electron Lasers
}

\author{
P. Emma ${ }^{\dagger}$ and G. Stupakov ${ }^{\ddagger}$ \\ Stanford Linear Accelerator Center, \\ Stanford University, Stanford, CA 94309
}

\begin{abstract}
Several ideas have been proposed to 'condition' an electron beam prior to the undulator of a Free-Electron Laser (FEL) by increasing each particle's energy in proportion to the square of its transverse betatron amplitude. This conditioning enhances FEL gain by reducing the axial velocity spread within the electron bunch. We demonstrate that for symplectic beamlines, and independent of the method, this conditioning is always accompanied by a large head-tail focusing variation which, for short wavelength FELs, is so severe as to make conditioning completely impractical. We furthermore find that any system added to correct the head-tail focusing variation will also remove the conditioning. As an example, a new method for conditioning is presented and shown to result in exactly the same head-tail focussing problem as that of reference [1].
\end{abstract}

\footnotetext{
$\dagger^{\dagger}$ Emma@SLAC.Stanford.edu

${ }^{\ddagger}$ Stupakov@SLAC.Stanford.edu
}

Presented at Particle Accelerator Conference (PAC 03), 5/12/2003-5/16/2003, Portland, OR, USA

Work supported by Department of Energy Contract DE-AC03-76SF00515. 


\section{INTRODUCTION}

The most demanding requirement for future Free-Electron Lasers (FELs) in the x-ray regime $[2,3]$ is the reliable generation of a sufficiently small transverse electron emittance. To mitigate this problem, several ideas have been proposed to 'condition' an electron beam prior to the undulator by increasing each particle's energy in proportion to the square of its betatron amplitude [1, 4-6]. This conditioning enhances FEL gain by reducing the axial velocity spread within the electron bunch generated over the undulator, due both to energy spread and finite transverse emittance. A common proposal for generating the conditioning correlation is to differentially accelerate off-axis particles with a series of $\mathrm{TM}_{210}$-like mode transverse RF structures. But the conditioning can also be accomplished by delaying large amplitude particles in a long, strong focussing channel and differentially accelerating the delayed particles with off crest-phase acceleration [6]. In reference [1] an undesirable sideeffect is briefly mentioned, due to the transverse time-dependent magnetic field, which results in a head-tail focusing variation along a bunch with finite length. A correction is also suggested using RF-quadrupoles. Unfortunately, as we show in this paper, this side-effect is a necessary outcome of conditioning, due to the symplecticity of the map describing the beamline, and it can be prohibitively large. In the conditioner at the end of reference [6], although not discussed there, the effect will appear as the chromaticity of a strong focusing channel on a chirped beam. Furthermore, we find that any system added to correct this head-tail focusing variation will necessarily remove the energy conditioning.

In this paper, we briefly review the requirements for FEL beam conditioning and, as an example, present a new conditioning scheme using strong solenoid magnets, which at first examination looks promising. But the head-tail focusing again appears and we quantify the resulting 'projected' (i.e., bunch-length integrated) transverse emittance growth, relating it directly to the FEL parameters. A numerical example is used to demonstrate the extreme severity of the effect and the impracticality of conditioning a short-wavelength FEL. We then present a general symplectic beam conditioner using generator functions and show the unavoidable relation between conditioning and projected transverse emittance growth. 
Finally, we discuss the limitations, provide a more familiar analogy, and summarize our results.

\section{FEL BEAM CONDITIONING}

Electron beam conditioning, as proposed in [1], increases each particle's energy in proportion to the square of its betatron amplitude. A particle with high energy travels a shorter path in an undulator (increased mean axial velocity), while a large betatron amplitude delays a particle by lengthening its path through the undulator [7]. The conditioning correlation establishes a cancellation of these two effects, resulting in a significant reduction of the axial velocity spread, enhancing the FEL gain, or perhaps allowing the FEL to operate with a larger transverse emittance than required without conditioning. The relative energy conditioning requirement, for natural undulator focusing, can be written as [1]

$$
\delta_{u}=\delta_{n}+\frac{1}{4 \gamma_{u}} \frac{\epsilon_{N}}{\beta_{u}} \frac{\lambda_{u}}{\lambda_{r}} r^{2},
$$

where $\delta_{n}\left(\ll \delta_{u}\right)$ is the non-conditioned component of the particle's relative energy deviation, $\gamma_{u}$ is the electron energy in the undulator (in units of rest mass), $\epsilon_{N}\left(=\gamma_{u} \epsilon\right)$ is the normalized rms transverse emittance (equal in $x$ and $y$ ), $\beta_{u}\left(=\beta_{x}=\beta_{y}\right.$ ) is the constant beta-function in the undulator, $\lambda_{u}$ is the undulator period, $\lambda_{r}$ is the FEL radiation wavelength, and $r$ is the invariant normalized $4 \mathrm{D}$ betatron amplitude of the particle,

$$
r^{2} \equiv \frac{x^{2}+\left(\beta_{u} x^{\prime}\right)^{2}+y^{2}+\left(\beta_{u} y^{\prime}\right)^{2}}{\beta_{u} \epsilon} .
$$

The index ' $u$ ' above indicates the value of the respective quantity in the undulator. The betatron amplitude, $r$, is expressed in terms of a particle's transverse positions, $x$ and $y$, and angles, $x^{\prime}$ and $y^{\prime}$, with natural focusing where $\alpha_{x}=\alpha_{y}=0$. A conditioner beamline is designed to imprint this $\delta_{u} \sim r^{2}$ correlation within the electron bunch, with coefficient given in Eq. (1).

In a general case, the conditioning might be performed at low energy near the injector where the bunch is still relatively long. For short wavelength FELs, the bunch is compressed and accelerated after the injector, and both effects scale the conditioning, but in the absence 
of particle mixing, do not alter its correlation character. Acceleration from $\gamma_{0}$ ('energy' at injector) to $\gamma_{u}$ ('energy' in undulator) reduces the conditioned relative energy spread, while

compression from an initial bunch-length, $\sigma_{z_{0}}$, to a shorter final bunch-length, $\sigma_{z_{f}}$, amplifies the conditioning. The latter is true because the bunch is shortened after compression and since the longitudinal emittance is preserved the energy spread must be increased proportionally, independent of the imprinted $r^{2}$-correlation. The relative energy deviation, $\delta$, at the location of the conditioner, before acceleration and compression, must then be scaled by the acceleration and compression factors:

$$
\delta=\frac{\sigma_{z_{f}}}{\sigma_{z_{0}}} \frac{\gamma_{u}}{\gamma_{0}}\left(\delta_{n}+\frac{1}{4 \gamma_{u}} \frac{\epsilon_{N}}{\beta_{u}} \frac{\lambda_{u}}{\lambda_{r}} r^{2}\right) .
$$

This means that, depending on the level of bunch compression, the relative energy spread immediately after a low-energy conditioner is not necessarily larger than that at the undulator.

\section{A ONE-PHASE SOLENOID CONDITIONER}

As an example beam conditioner, and to show the ultimate limitations of conditioners, we describe here a simplified system composed of a solenoid magnet and RF accelerating sections. Its specific limitations, however, will be completely applicable to all conditioners which are based on differential acceleration of off-axis particles. The limitations can be described in terms of an effective transverse emittance growth and this growth will be related only to the FEL conditioning requirements, and not to the specific method of conditioning.

The conditioner is shown in Fig. 1, and is composed of a solenoid magnet sandwiched between two RF accelerating sections operated at opposing zero-crossing phases. (A similar idea was proposed at the end of reference [6].) The first RF section 'chirps' the energy along the bunch length, and the final section removes the chirp. The conditioning is generated in the solenoid by the delay of particles with large amplitudes in $x$ and $y$. The solenoid strength is set to produce a $+\mathbf{I}$ linear transfer matrix in $6 \mathrm{D}$ with the relation $|k| L=n \pi$ $(n=1,2,3, \ldots)$, where $k \equiv \frac{1}{2} B_{z} /(B \rho), L$ is the solenoid length, $B_{z}$ is its axial magnetic field, and $(B \rho)$ is the standard magnetic rigidity $\left(=p_{0} / e\right)$. The particle coordinates within 


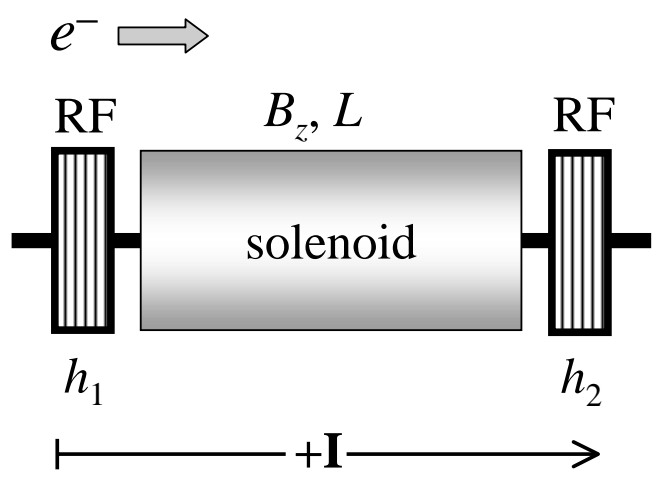

FIG. 1: One-phase electron beam conditioner composed of solenoid magnet sandwiched between RF accelerating sections operated at opposing zero-crossing phases.

the bunch at the entrance to the system are $\left(x_{0}, x_{0}^{\prime}, y_{0}, y_{0}^{\prime}, z_{0}, \delta_{0}\right)$, where $\delta_{0} \equiv \Delta p / p_{0}$, and we assume these variables are initially uncorrelated and have zero mean. For simplicity, we use a cylindrically symmetric beam with initial Twiss parameters: $\beta_{x}=\beta_{y}=\beta$, and $\alpha_{x}=\alpha_{y}=0$. The Twiss parameters are unchanged, to $1^{\text {st }}$-order, across the solenoid and across each 'thin' RF section. The electrons are also assumed to be ultra-relativistic.

The first RF section changes the relative energy deviation of a particle to: $\delta_{1}=\delta_{0}+h_{1} z_{0}$, where $h_{1}$ is the linear RF-induced slope $(h \equiv d \delta / d z)$. For simplicity, the RF sections are treated as thin elements which do not alter the transverse coordinates. After the solenoid, the coordinates are unchanged to $1^{\text {st }}$-order, but a chromatic $2^{\text {nd }}$-order aberration is added to the angles with $\Delta x^{\prime}=2 T_{216} x_{0}\left(\delta_{0}+h_{1} z_{0}\right)$ and $\Delta y^{\prime}=2 T_{436} y_{0}\left(\delta_{0}+h_{1} z_{0}\right)$. All other $2^{\text {nd }}$-order transverse aberrations are small in comparison for the case: $|k| \beta \gg 1,|k| L=n \pi[8]$.

The energy is not changed in the solenoid, but the longitudinal coordinate is delayed by the helical trajectory according to $z_{1}=z_{0}+T_{511} x_{0}^{2}+T_{533} y_{0}^{2}$ (bunch head at $z>0$ ). Similarly, all other $2^{\text {nd }}$-order longitudinal aberrations are small for the case $|k| \beta \gg 1$. The $2^{\text {nd }}$-order coefficients of a solenoid with $|k| L=n \pi$ are related to each other by: $T_{511}=$ $T_{533}=-T_{216}=-T_{436}=-k^{2} L / 2[8]$, which, as shown in section $\mathrm{V}$, is an unavoidable connection for symplectic systems. The final RF section, $h_{2}$, changes the energy according to $\delta=\delta_{1}+h_{2} z_{1} \approx\left(h_{1}+h_{2}\right) z_{0}-\frac{1}{2} k^{2} L h_{2}\left(x_{0}^{2}+y_{0}^{2}\right)$. The second chirp is chosen equal and opposite to the first, $h_{1}=-h_{2} \equiv h$, and the final coordinate map across the conditioner, to 
second order and for $\left|\delta_{0}\right| \ll\left|h z_{0}\right|$, becomes

$$
\begin{aligned}
x & =x_{0}, \\
x^{\prime} & =x_{0}^{\prime}+k^{2} L h z_{0} x_{0}, \\
z & =z_{0}-\frac{1}{2} k^{2} L\left(x_{0}^{2}+y_{0}^{2}\right), \\
\delta & =\delta_{0}+\frac{1}{2} k^{2} \operatorname{Lh}\left(x_{0}^{2}+y_{0}^{2}\right),
\end{aligned}
$$

with similar relations in $y$ and $y^{\prime}$. The final energy deviation, $\delta$, is clearly conditioned (for $h>0$ ) in both planes but in only one betatron phase (i.e., $x_{0}$, but not $x_{0}^{\prime}$ ). This system provides spatial (but not angular) conditioning described by

$$
\delta=\delta_{0}+\frac{1}{2} k^{2} L h \beta \epsilon_{0} r^{2}, \quad r^{2} \equiv \frac{x_{0}^{2}+y_{0}^{2}}{\beta \epsilon_{0}} .
$$

Two solenoids can also be used, separated by a $\pi / 2$-transformer to condition both betatron phases, but here we simplify the description by considering only a one-phase conditioner.

The bunch-length coordinate, $z$, in Eqs. (4) also includes a non-linear distortion due to the solenoid delay of large amplitude particles. This can easily be removed, without

changing the energy conditioning, by adding a four-dipole chicane, or similar section, with $R_{56}=1 / h>0$, after the final RF section, resulting in $z=z_{0}+R_{56} \delta_{0} \approx z_{0}$. The bunch is then restored to its initial length with no significant change in distribution. This point is not relevant here, so we ignore this correctable distortion and instead examine the energy conditioning and the associated transverse aberrations.

\section{ENERGY CONDITIONING AND TRANSVERSE EMITTANCE GROWTH}

The conditioning coefficient in Eq. (3) can be equated to that in Eq. (5) producing the conditioning requirement for the solenoid system

$$
k^{2} L h \beta \sigma_{z_{0}}=\frac{1}{2} \frac{\lambda_{u}}{\lambda_{r}} \frac{\sigma_{z_{f}}}{\beta_{u}} \equiv a,
$$

where the solenoid-conditioner parameters are on the left side and the FEL parameters are on the right, and here we define the dimensionless conditioning coefficient, $a$. In the typical 
case of a short wavelength FEL, the conditioning parameter $a$ is large, $a \gg 1$, (see numerical example below).

The chirp parameter, $h$, is more easily interpreted by relating it to the rms relative energy spread in the solenoids: $\sigma_{\delta_{1}} \approx|h| \sigma_{z_{0}}$. This also makes it clear that the transverse aberrations in Eqs. $(4)$ are chromatic $\left(\delta_{1} \approx h z_{0}\right)$, and it is useful at this point to quantify the aberrations in terms of an effective transverse emittance growth. The rms emittance after the solenoid is calculated using the first two lines of Eqs. (4) and the first and second moments of the particle ensemble:

$$
\epsilon_{x}^{2}=\left\langle(x-\bar{x})^{2}\right\rangle\left\langle\left(x^{\prime}-\overline{x^{\prime}}\right)^{2}\right\rangle-\left\langle(x-\bar{x})\left(x^{\prime}-\overline{x^{\prime}}\right)\right\rangle^{2}
$$

The mean values, $\bar{x}=\langle x\rangle$, and $\overline{x^{\prime}}=\left\langle x^{\prime}\right\rangle$ are zero since the initial coordinates are uncorrelated and have zero mean. The correlation $\left\langle x x^{\prime}\right\rangle$ is zero for the same reasons, so the $x$-emittance after the solenoid is:

$$
\begin{aligned}
\epsilon_{x}^{2} & =\left\langle x^{2}\right\rangle\left\langle x^{\prime 2}\right\rangle \\
& \approx\left\langle x_{0}^{2}\right\rangle\left\langle\left(x_{0}^{\prime}+k^{2} L h z_{0} x_{0}\right)^{2}\right\rangle \\
& =\epsilon_{x 0}^{2}\left[1+\left(k^{2} L h \beta \sigma_{z_{0}}\right)^{2}\right]
\end{aligned}
$$

where $\left\langle x_{0}^{2}\right\rangle=\beta \epsilon_{x 0},\left\langle x_{0}^{\prime 2}\right\rangle=\epsilon_{x 0} / \beta$, and $\left\langle z_{0}^{2}\right\rangle=\sigma_{z_{0}}^{2}$, with a similar form in $y$. The relative emittance growth after the solenoid is

$$
\frac{\epsilon_{x}}{\epsilon_{x 0}} \approx k^{2} L h \beta \sigma_{z_{0}}=a \gg 1
$$

which is identical to the conditioning relation in Eq. (6), providing a direct connection between transverse emittance growth and FEL conditioning requirements.

For parameters of the LCLS [2] shown in Table I (using a beta function for natural focusing, to be consistent with Eq. 1), the relative emittance growth is extremely large at $\epsilon_{x} / \epsilon_{x 0} \approx 33$. The parameters for the VISA FEL [9] are also included showing that conditioning may still be possible at longer wavelengths.

This growth is actually an increase of the 'projected' transverse emittance integrated over the bunch length. The second line of Eq. (4) shows that the bunch head $\left(z_{0}>0\right)$ is defocused (equating: $\left.k^{2} L h z_{0}=1 / f\right)$, while the bunch tail $\left(z_{0}<0\right)$ is focused. The significance 
TABLE I: FEL and conditioner parameters for the LCLS [2] and VISA [9].

\begin{tabular}{|l|c|c|c|c|}
\hline parameter & symbol & LCLS & VISA & units \\
\hline electron energy $/ m c^{2}$ in undulator & $\gamma_{u}$ & 28000 & 140 & \\
\hline undulator period & $\lambda_{u}$ & 3 & 1.8 & $\mathrm{~cm}$ \\
\hline radiation wavelength & $\lambda_{r}$ & 1.5 & 8500 & $\AA$ \\
\hline und. beta-function (natural focusing) & $\beta_{u}$ & 72 & 0.6 & $\mathrm{~m}$ \\
\hline final rms bunch length & $\sigma_{z_{f}}$ & 24 & 100 & $\mu \mathrm{m}$ \\
\hline conditioning coefficient & $a$ & 33 & 1.8 & \\
\hline
\end{tabular}

of the focusing variation is evaluated by comparing the focal length, $f$, at $z_{0}= \pm \sigma_{z_{0}}$, to $\beta$. The ratio is $\beta / f\left( \pm \sigma_{z_{0}}\right)= \pm k^{2} L \beta h \sigma_{z_{0}}= \pm a$, which is a very strong effect $(\beta /|f| \gg 1)$ for short wavelength FELs, and is precisely the conditioning coefficient given in Eqs. (6).

With a chirped energy spread, the chromatic effects of the solenoid are equivalent to the effects of an RF-quadrupole (RFQ). It is interesting to compare this result with that of reference [1], where a completely different conditioner beamline, employing transverse RF cavities, produced an undesirable RFQ effect. In fact, as shown in the next section, FEL beam conditioning in a symplectic beamline always produces an undesirable RFQ-effect, which is extremely large for short wavelength FELs, as given in Eq. (??).

\section{A GENERAL CONDITIONER}

In this section we will show that the transverse emittance growth associated with conditioning is not related to the specific design outlined in the previous section, but is a general feature of any conditioner, and is due to the symplecticity of the map between the entrance to and exit from the conditioner.

To simplify consideration, we assume that the conditioner does not introduce coupling between the vertical and horizontal planes, and consider only the horizontal plane with the initial values of coordinates $\left(x_{0}, x_{0}^{\prime}\right)$ at the entrance, and the final values $\left(x, x^{\prime}\right)$ at the exit. Consideration of the vertical coordinates $y, y^{\prime}$ can be carried out analogously to $x, x^{\prime}$. We 
will also assume that the initial and final values of the longitudinal coordinate are the same: $z=z_{0}$. Instead of using the variables $x_{0}, x_{0}^{\prime}$ and $x, x^{\prime}$, it is convenient and more general to introduce new variables $u_{0}, v_{0}$, and $u, v$, such that

$$
\left(\begin{array}{l}
u_{0} \\
v_{0}
\end{array}\right)=Q_{0}\left(\begin{array}{l}
x_{0} \\
x_{0}^{\prime}
\end{array}\right), \quad\left(\begin{array}{l}
u \\
v
\end{array}\right)=Q\left(\begin{array}{l}
x \\
x^{\prime}
\end{array}\right),
$$

where the matrices $Q_{0}$ and $Q$ are

$$
Q_{0}=\frac{1}{\sqrt{\beta_{0}}}\left(\begin{array}{cc}
1 & 0 \\
\alpha_{0} & \beta_{0}
\end{array}\right), \quad Q=\frac{1}{\sqrt{\beta}}\left(\begin{array}{cc}
1 & 0 \\
\alpha & \beta
\end{array}\right),
$$

with $\beta_{0}, \alpha_{0}$ and $\beta, \alpha$ the Twiss parameters at the entrance and exit of the conditioner, respectively. Being symplectic linear transformations, $Q$ and $Q_{0}$ conserve the symplecticity of the map from $\left(u_{0}, v_{0}\right)$ to $(u, v)$. Note, that in linear approximation this map has a form

$$
\left(\begin{array}{l}
u \\
v
\end{array}\right)=A\left(\begin{array}{l}
u_{0} \\
v_{0}
\end{array}\right)
$$

where

$$
A=\left(\begin{array}{cc}
\cos \psi & \sin \psi \\
-\sin \psi & \cos \psi
\end{array}\right)
$$

with $\psi$ the betatron phase advance across the conditioner. Also note that the contribution of the $x$-coordinate $x_{0}^{2} /\left(\beta \epsilon_{0}\right)$ to the parameter $r$ in Eq. (5) is equal to $u_{0}^{2} / \epsilon_{0}$, and the conditioning requirement Eq. (5) can be written as

$$
\delta=\delta_{0}+\frac{1}{2} b u_{0}^{2}
$$

where $b=a / \sigma_{z_{0}}$, and the conditioning constant $a$ is given by Eq. (6).

To derive a general symplectic map which in linear approximation reduces to the linear map Eq. (13) and also includes the conditioning given by Eq. (14), we will use a method of generating functions [10]. We choose a generating function which depends on initial coordinates $u_{0}$ and $z_{0}$ and final momenta $v$ and $\delta, F\left(u_{0}, z_{0}, v, \delta\right)$. The map is defined by the relations

$$
v_{0}=\frac{\partial F}{\partial u_{0}}, \quad \delta_{0}=\frac{\partial F}{\partial z_{0}}, \quad u=\frac{\partial F}{\partial v}, \quad z=\frac{\partial F}{\partial \delta}
$$


In paraxial approximation all coordinates and momenta are considered small and we can expand $F$ in a Taylor series. The linear terms in this expansion vanish because zero initial coordinates and momenta map to zero final ones. The expansion begins from the second order terms

$$
F \approx F_{2}+F_{3}+\ldots
$$

where $F_{2}$ is a quadratic, and $F_{3}$ is a cubic function of the coordinates and momenta. The function $F_{2}$ should generate the linear map Eq. (13) for $u$ and $v$ with a unit transformation for $z$ and $\delta$ - a direct calculation shows that

$$
F_{2}=\frac{1}{2}\left(u_{0}^{2}+v^{2}\right) \tan \psi+u_{0} v \sec \psi+\delta z_{0}
$$

The function $F_{3}$ generates $2^{\text {nd }}$-order abberations in the system, out of which we choose only a term responsible for the conditioning:

$$
F_{3}=-\frac{1}{2} b z_{0} u_{0}^{2}
$$

Indeed, using the second of Eqs. (15) with Eqs. (17) and (18) we find

$$
\delta_{0}=\delta-\frac{1}{2} b u_{0}^{2}
$$

in agreement with Eq. (14). At the same time the first and the third of Eqs. (15) yield

$$
\begin{aligned}
& v_{0}=u_{0} \tan \psi+v \sec \psi-b z_{0} u_{0} \\
& u=v \tan \psi+u_{0} \sec \psi
\end{aligned}
$$

These equations can be easily solved for $u$ and $v$ :

$$
\begin{aligned}
& u=u_{0} \cos \psi+v_{0} \sin \psi+b z_{0} u_{0} \sin \psi, \\
& v=-u_{0} \sin \psi+v_{0} \cos \psi+b z_{0} u_{0} \cos \psi .
\end{aligned}
$$

We emphasize here that the same term in the symplectic map Eq. (18) that is responsible for the conditioning of the beam also introduces in Eq. (22) the transverse deflection that varies along the bunch. This also means that adding a system that 'fixes' this deflection downstream of the conditioner would inevitably remove the conditioning itself. 
Finally, we will calculate the emittance increase of the beam due to the conditioning. Using Eq. (7) for the emittance, with $\bar{u}=\bar{v}=0$, we find

$$
\epsilon_{x}^{2}=\left\langle u^{2}\right\rangle\left\langle v^{2}\right\rangle-\langle u v\rangle^{2}
$$

Substituting the map, Eqs. (22), into this yields

$$
\epsilon_{x}^{2}=\epsilon_{x 0}^{2}\left(1+b^{2} \sigma_{z_{0}}^{2}\right)=\epsilon_{x 0}^{2}\left(1+a^{2}\right)
$$

in agreement with Eq. (8), but now in a general case with arbitrary phase advance, $\psi$, and non-zero initial alpha function, $\alpha_{0}$. For the specific conditioner described in Section III, we have $\psi=2 n \pi, \beta_{0}=\beta, \alpha_{0}=\alpha=0$, and Eqs. (22) reproduce the first two of Eqs. (4).

\section{DISCUSSION}

We have demonstrated for a general one-phase conditioner that a strong head-tail focusing variation will always accompany the energy conditioning correlation, and that this focusing variation is set solely by the FEL parameters, and not the conditioner. An analogous situation can be described by considering the addition of a skew-quadrupole to an uncoupled transport line. If the desired effect (the 'conditioning') is an $\left\langle x^{\prime} y\right\rangle$ correlation, the skewquadrupole will suffice, but an accompanying $\left\langle x y^{\prime}\right\rangle$ correlation will also result and may be an extremely important side-effect, depending on the $x$-to- $y$ emittance ratio. The two effects are, of course, scaled by the same skew-quadrupole strength and are not independent. This example is a linear one, while energy conditioning is $2^{\text {nd }}$-order. However, both cases are based on symplectic transformations including coupling between two planes.

A two-phase conditioner is more complicated, but does not qualitatively change the arguments presented here. If a second solenoid is added to Fig. 1, with a $\pi / 2$-transformer separating it from the first solenoid, a two-phase conditioner is formed. The chromatic aberrations, however, generated in the first solenoid will distort the beam in the second solenoid and the energy conditioning will then include large terms higher than $2^{\text {nd }}$-order, which will likely dominate and spoil the conditioning. A two-phase system will then only magnify the projected emittance growth, and also degrade the character of the conditioning itself. 
The authors have studied various forms of two-phase conditioners and, while not presenting a rigorous proof of it here, find the emittance growth much larger than a one-phase system. An analytical calculation requires care in retaining the symplectic map to very high order. Tracking the LCLS case through the two-phase solenoid system shows a projected transverse emittance growth of $\epsilon_{x} / \epsilon_{x 0}>10^{4}$. We believe this result will be the same for any symplectic energy conditioner designed for the LCLS parameters.

Finally, conditioning is even less practical for an undulator with strong FODO-cell focusing [7]. In this case, the path length increase with increased betatron amplitude [see Eq. (1)] is nearly twice that of natural focusing, and the energy spread necessary for conditioning is then twice larger. This effect, in conjunction with the reduced mean beta function, $\beta_{u}$, in a FODO-cell undulator, makes the conditioning coefficient, $a$, and therefore the projected transverse emittance growth, even larger than for natural focusing.

For these reasons, we conclude that conditioning by adding an energy correlation with the square of the betatron amplitude is impractical if not impossible in short wavelength FELs.

\section{ACKNOWLEDGMENTS}

This work was supported by the Department of Energy, contract DE-AC03-76SF00515. We also thank Karl Bane, Max Cornacchia, Claudio Pellegrini, Jim Welch, and Juhao Wu for useful discussions.

\section{REFERENCES}

[1] A. Sessler et al., Phys. Rev. Lett., 68, 309 (1992).

[2] LCLS CDR, SLAC Report No. SLAC-R-593, 2002.

[3] TESLA TDR, DESY Report No. DESY-2001-011, 2001.

[4] P. Sprangle et al., Nucl. Inst. and Meth. A 331 (1993) 6.

[5] H. Liu and G. R. Neil, in Proc. of the PAC'93, Washington, DC (IEEE, Piscataway, NJ, 1993), p. 279. 
[6] N. A. Vinokurov, Nucl. Inst. and Meth. A 375 (1996) 264.

[7] S. Reiche, Nucl. Inst. and Meth. A 445 (2000) 90.

[8] F. C. Iselin, The MAD Program Ver. 8.13, Physical Methods Manual (1994), p. 29.

[9] A. Tremaine et al., in Proc. of the PAC'01, Chicago, IL (IEEE, Piscataway, NJ, 2001), p. 2760 .

[10] H. Goldstein, Classical Mechanics, (Addison-Wesley, London, 1980). 